\title{
A SIMPLE SELLING AND BUYING PROCEDURE ${ }^{*}$
}

\author{
David Pérez-Castrillo ${ }^{+}$ \\ Universitat Autònoma de Barcelona \\ Marilda Sotomayor ${ }^{++}$ \\ Universidade de Sao Paulo
}

\begin{abstract}
For the assignment game, we analyze the following mechanism: sellers, simultaneously, fix their prices first; then buyers, sequentially, decide which object to buy, if any, among the remaining objects. The first phase of the game determines the potential prices, while the second phase determines the actual matching. We prove that the set of subgame perfect equilibria in pure strategies in the strong sense of the mechanism coincides with the set of sellers' optimal stable outcomes when buyers use maximal strategies. That is, the mechanism leads to the maximum equilibrium prices and to an optimal matching.
\end{abstract}

JEL numbers: C78, D78.

Keywords: Matching model, Assignment model, Mechanism, Implementation.

We thank Carmen Beviá, Jordi Massó, and Alvin Roth for helpful comments. Pérez-Castrillo also acknowledges the financial support from projects DGES PB 92-0590, DGES PB 96-1192, and SGR 9862. Sotomayor acknowledges J.S. Guggenheim foundation, FAPESP, and FIPE - Sao Paulo - Brazil. She also acknowledges the hospitality of the Universitat Autònoma de Barcelona and University of Pittsburgh, where part of this work has been done.

+ Universitat Autònoma de Barcelona

Departament d'Economia i d'Història Econòmica \& CODE

08190 Bellaterra, Barcelona; Spain

e-mail: David.Perez@uab.es

++ Universidade de Sao Paulo

Departamento de Economia

Av. Prof. Luciano Gualberto, 908

Cidade Universitária

05508 - 900 Sao Paulo, SP, Brazil

e-mail: marildas@usp.br 


\section{1- INTRODUCTION}

We consider markets where a finite number of sellers and buyers meet, with the particularity that each seller only owns one object and each buyer only wants to buy, at most, one object. These markets are usually referred to as the assignment game, which was introduced by Shapley and Shubik (1972). Buyers are heterogeneous in their preferences about the different objects. Associated with each possible partnership there is a non-negative real number that represents the worth of the partnership. An outcome of this game specifies a matching between buyers and sellers and the price that each buyer pays to the owner of the object she is buying.

The solution concept usually considered to analyze these markets is stability. An outcome is stable if it is individually rational and there is no partnership and a price so that, at this price, both buyer and seller are better off under this partnership than under the previous outcome. Shapley and Shubik (1972) show that the set of stable allocations is non-empty and it is a complete distributive lattice. This set contains a special allocation giving an optimal payoff to the buyers (which is the worse for the sellers) and another one giving an optimal payoff to the sellers (the worst for the buyers). Moreover, the set of stable allocations coincides with the set of competitive equilibria, the two extreme allocations corresponding to the minimum and to the maximum equilibrium prices, respectively.

The assignment game allows for the analysis of markets with a finite number of heterogeneous sellers and buyers. The participants in these markets typically take decisions strategically. Each seller determines the price for his object looking for the maximum revenue, taking into account the prices posted by the other sellers. Similarly, each buyer tries to buy the best available object, given the prices. A natural question is then whether the non-cooperative (strategic) behavior by sellers and buyers actually leads to outcomes that are stable, or competitive.

We analyze the following mechanism, which tries to capture the relevant interaction among sellers and buyers: sellers, simultaneously, fix their prices first; then buyers, sequentially, decide which object to buy, if any, among the remaining objects. The first 
phase of the game determines the potential prices, while the second phase determines the actual matching. The mechanism is very simple in terms of strategies: each seller only proposes a price for his object, each buyer only chooses an object. Also, the decisions by the buyers directly provide a matching, the decisions by the sellers are the final prices for the objects sold, and there is no need for a referee.

We look for the subgame perfect equilibria in pure strategies (SPE) of the previous mechanism. We restrict attention to a certain class of strategies of the buyers, that we call maximal strategies (the matchings induced by these strategies are called maximal matchings). To explain the meaning of a maximal strategy, consider a situation with two buyers, Alph and Bob, and two objects. Suppose that the prices have already been set and that Alph is indifferent between the two objects. However, if she chooses the first object then Bob obtains a high utility by buying the second one, while if Alph chooses the second object then Bob does not want to buy the other. We say that Alph buying the first object (and Bob the second) is a maximal strategy, while Alph buying the second object (and Bob not buying) is not. Notice that both strategies are SPE of the game that starts once the prices have been decided. A matching is maximal if it is Pareto efficient for the buyers among the matchings that result as SPE of the second phase of the mechanism.

We also concentrate on the analysis of the SPE in the strong sense (see Demange and Gale (1985)). To be equilibrium in the strong sense, the strategies of the participants must be robust to deviations by any optimistic seller. More precisely, we assume that a seller increases his price whenever there is a maximal matching for the buyers where his object is actually sold.

We prove that, when buyers use maximal strategies, the set of SPE in the strong sense of the proposed mechanism coincides with the set of sellers' optimal stable outcomes. That is, strategic behavior by sellers and buyers in the assignment game leads to (the maximum) equilibrium prices and to an optimal matching for a mechanism that, we think, captures the main ingredients of the interaction among sellers and buyers in many markets. 
The implementation result conveys interesting information about the properties of the outcome of the mechanism. The first property is that the set of SPE in the strong sense, when buyers use maximal strategies, does not depend on the order of the buyers, that is, the order of the line does not matter in equilibrium. The second property is that, in equilibrium, every buyer is buying an object that is optimal for her given the prices. In equilibrium, every buyer obtains an object in her demand set and each seller willing to sell can do so. Moreover, no seller has an incentive to choose a different price.

In this paper, we follow the line of research initiated by Gale and Shapley (1962) in their seminal paper. They introduce the deferred acceptance algorithm for the marriage problem. In this mechanism, each $\operatorname{man}^{1}$ proposes to his favorite woman, if she is acceptable to him. Each woman accepts the most preferred man among the offers she receives, if he is acceptable to her. Accepted men remain provisionally engaged, while rejected men can make new proposals to their next choice. The algorithm stops at the first step in which no man is rejected.

Gale and Shapley (1962) show that when participants declare their true preferences the matching produced by this simple and nice algorithm is an allocation that all men prefer to any other stable allocation. ${ }^{2}$ Even when the participants can act strategically, the outcome is still nice: truthful revelation of preferences is a dominant strategy for men (Dubins and Freedman (1981) and Roth (1982)) and the equilibrium of the game where the men state their true preferences and women can choose any preference is still an stable allocation (Roth, 1984). Moreover, Gale and Sotomayor (1985) show that the women's optimal stable allocation is the strong equilibrium of the game, when men play their dominant strategy.

Besides the analysis of the deferred acceptance algorithm, several authors have looked for other simple mechanisms that lead to stable allocations for different matching models. For the marriage problem, Alcalde (1996) presents a mechanism, close to that

1 The mechanism can also be implemented exchanging the roles of men and women. 
of Gale and Shapley, which implements the correspondence of stable matchings in undominated equilibria. Alcalde, Pérez-Castrillo, and Romero-Medina (1998), and Alcalde and Romero-Medina (1999) implement through simple mechanisms the set of stable matchings in the college-admissions problem and in the job matching market, respectively. Moreover, they also implement particular subsets of the stable correspondence. ${ }^{3}$

For the assignment game, Demange and Gale (1985) analyze the properties of a mechanism in which agents announce their demand and supply functions, and then a referee calculates the minimum equilibrium price and allocates the objects accordingly. However, the mechanism is manipulable for the sellers: they can lead the payoff to the maximum rather than the minimum equilibrium price by falsifying their supply functions. In the same framework, Demange, Gale, and Sotomayor (1986) propose two dynamic auction mechanisms, although they do not analyze the possibility of manipulative behavior.

Also for the assignment game, Kamecke (1989) analyzes the strategic interaction between buyers and sellers in a mechanism that shares common features to ours. He considers the following game: First, sellers announce their payoff claims. Then one buyer after the other addresses her demand and chooses a seller. Finally, sellers select again one of their potential customers. For matched couples, the agents get what they asked for if their two claims are feasible. Also, the payoff function assigns to a seller the payoff that was offered to him if it exceeds his claim. Additionally, agents pay a positive cost if they address demands without being successful. This mechanism implements in SPE the seller-optimal stable payoff. ${ }^{4}$ The nice feature of Kamecke's result is that it

2 In the same spirit, Crawford and Knoer (1981) and Kelso and Crawford (1982) introduce a salaryadjustment process for the job matching market.

3 For general cooperative games in characteristic form, several papers have recently addressed the question of implementation of the core (Pérez-Castrillo (1994), Perry and Reny (1994), Serrano (1995)).

4 Kamecke (1989) also analyzes a mechanism similar to the one we have presented but in which demands by sellers and claims and choice of opponent by buyers are all made simultaneously at the first period. He shows that this mechanism implements in SPE the set of stable allocations. 
does not need additional considerations concerning sellers' and buyers' strategies. However, the mechanism that we propose is simpler, closer to the functioning of the markets, and it does not need some (third) party to allocate the surplus of a partnership, if any, and to enforce the payment of unsuccessful demands. Note that both mechanisms share the interesting property that they provide a strong first mover advantage: the fact that sellers fix their prices first makes it that, at equilibrium, the final prices are the highest among the competitive prices.

The paper is organized as follows. In Section 2, we present the cooperative model of the assignment game. In Section 3, we describe the mechanism proposed. In Section 4, we analyze the set of equilibria in the strong sense when buyers use maximal strategies and we state the main results. In Section 5 we discuss the use of maximal strategies, while in Section 6, we present some examples of possible outcomes of the mechanism when we do not restrict attention to maximal strategies or to equilibria in the strong sense. In Section 7, we conclude. Finally, an Appendix contains some of the proofs.

\section{2- THE COOPERATIVE MODEL}

We consider a buyer-seller market in which each seller owns only one indivisible object and each buyer wants at most one of those objects. Generic buyers will be denoted by $p_{i}$ and $p_{k}$ and generic sellers by $q_{j}$ and $q_{h}$. The object owned by seller $q_{j}$ will also be denoted by $q_{j}$. Let $P=\left\{p_{1}, \ldots, p_{m}\right\}$ be the set of buyers and $Q=\left\{q_{0}, q_{1}, \ldots, q_{n-1}\right\}$ be the set of objects, where $q_{0}$ is an artificial "null object" that is introduced for technical convenience. More than one buyer may buy the object $q_{0}$. This convention allows us to treat a buyer $p_{i}$ that does not buy any object as if she bought the null object $q_{0}$.

For each pair $\left(p_{i}, q_{j}\right) \in P \times Q$ there is a non-negative number $\alpha_{i j}$ which can be interpreted as the maximum price that buyer $p_{i}$ is willing to pay for the object $q_{j}$. We will denote by $\alpha$ the $m \mathrm{x} n$ matrix $\left(\alpha_{i j}\right)_{i=1, \ldots, m ; j=0,1, \ldots, n-1}$. The value $\alpha_{i 0}$ is zero to all buyers. For simplicity, we assume that the reservation price of each seller is zero and that there are no monetary transfers among agents from the same side. Thus, if buyer $p_{i}$ buys the object $q_{j}$ at a price $v_{j}$ then the resulting utilities are $u_{i}=\alpha_{i j}-v_{j}$ for the buyer and $v_{j}$ for the 
seller. The price of the object $q_{0}$ is always zero, $v_{0}=0$, hence if buyer $p_{i}$ buys $q_{0}$ she obtains a utility $u_{i}=\alpha_{i 0}-v_{0}=0$. The buyer-seller market is denoted by $M \equiv(P, Q, \alpha)$.

Definition 1- A feasible matching $\mu$ for $M$ is a function from $P \cup Q-\left\{q_{0}\right\}$ onto $P \cup Q$ such that:

(a) for any $p_{i} \in P, \mu\left(p_{i}\right) \in Q$;

(b) for any $q_{j} \in Q-\left\{q_{0}\right\}$, either $\mu\left(q_{j}\right) \in P$ or $\mu\left(q_{j}\right)=q_{j}$;

(c) for any $\left(p_{i}, q_{j}\right) \in P \times Q-\left\{q_{0}\right\}, \mu\left(p_{i}\right)=q_{j}$ if and only if $\mu\left(q_{j}\right)=p_{i}$.

If $\mu\left(p_{i}\right)=q_{0}$, the buyer $p_{i}$ will also be called unmatched. If $\mu\left(q_{j}\right)=q_{j}$, the seller $q_{j}$ will be called unmatched (or the object $q_{j}$ will be called unsold). Given a set $A \subseteq P$, we denote $\mu(A) \equiv\left\{\mu\left(p_{i}\right) ; p_{i} \in A\right\}$.

Definition 2- A feasible matching $\mu$ is optimal for $M$ if for all feasible matching $\mu$ ':

$$
\sum_{\substack{p_{i} \in \mathcal{P} \\ q_{i}=\mu_{\left(p_{i}\right)}}} \alpha_{i j} \geq \sum_{\substack{p_{i} \in \mathcal{R} \\ q_{j}=\mu^{\prime}\left(p_{i}\right)}} \alpha_{i j}
$$

We denote by $R^{n}{ }_{+}$the set of vectors in $R^{n}$ with non-negative coordinates.

Definition 3- A feasible outcome for $M$, denoted by $(u, v ; \mu)$, is a pair of vectors $u \in R^{m}{ }_{+}$ and $v \in R^{n}{ }_{+}$and a feasible matching $\mu$ such that, for all $\left(p_{i}, q_{j}\right) \in P \times Q, u_{i}+v_{j}=\alpha_{i j}$ if $\mu\left(p_{i}\right)=$ $q_{j}$

Thus a feasible outcome may have unsold objects with price greater than zero. If $(u$, $v ; \mu)$ is a feasible outcome then $(u, v)$ is called a feasible payoff. The matching $\mu$ is said to be compatible with $(u, v)$ or with the prices $v$ and vice-versa. The vector $u$ will be called the payoff vector of the buyers associated to $(\nu, \mu)$.

Definition 4- Given the prices $v \in R^{n}{ }_{+}$and a matching $\mu$, compatible with $v$, we say that an object $q_{j}$ is $\mu$-expensive under $v$ if it is unsold under $\mu$, at a price $v_{j}>0$. 
Definition 5- A feasible outcome $(u, v ; \mu)$ is stable (or the payoff $(u, v)$ with the matching $\mu$ is stable) if $u_{i}+v_{j} \geq \alpha_{i j}$ for all $\left(p_{i}, q_{j}\right) \in P \times Q$ and there is no $\mu$-expensive object under $v$.

If $u_{i}+v_{j}<\alpha_{i j}$ for some pair $\left(p_{i}, q_{j}\right)$ we say that $\left(p_{i}, q_{j}\right)$ blocks the outcome $(u, v ; \mu)$ or the payoff $(u, v)$.

In the matching models treated here, the concept of stability is equivalent to the concept of the core. Moreover, it is possible to establish a relationship between stable outcomes and competitive equilibria of these markets.

Given $v \in R^{n}{ }$, the demand set of a buyer $p_{i}$ at prices $v$, denoted by $D_{i}(v)$, is the set of all objects which maximize $p_{i}$ 's utility payoffs. That is:

$$
D_{i}(v)=\left\{q_{j} \in Q ; \alpha_{i j}-v_{j} \geq \alpha_{i h}-v_{h} \text { for all } q_{h} \text { in } Q\right\} .
$$

The set $D_{i}(v)$ is always non-empty, since buyer $p_{i}$ has always the option of buying $q_{0}$. Also notice that, given $v$, buyer $p_{i}$ is indifferent about buying any object in $D_{i}(v)$.

Definition 6- The price vector $v \in R^{n}{ }_{+}$is called competitive if there exists a matching $\mu$ such that $\mu\left(p_{i}\right) \in D_{i}(v)$, for all $p_{i}$ in $P$. A matching $\mu$ such that $\mu\left(p_{i}\right) \in D_{i}(v)$ for all $p_{i}$ in $P$ is said to be competitive for the prices $v$.

Therefore, at competitive prices $v$, each buyer can be matched to an object in her demand set. There may be more than one competitive matching for the same price vector $v$.

Definition 7- The pair $(v, \mu)$ is a competitive equilibrium if $v$ is competitive, $\mu$ is competitive for $v$, and if $v_{j}=0$ for any unsold object $q_{j}$.

Thus, at a competitive equilibrium $(v, \mu)$, not only does every buyer get an object in her demand set, but there is no $\mu$-expensive object under $v$. If $(v, \mu)$ is a competitive equilibrium, $v$ will be called an equilibrium price vector. Clearly, to each competitive 
equilibrium $(v, \mu)$ we can associate a stable outcome $(u, v ; \mu)$ and vice-versa, by setting $u_{i}=\alpha_{i j}-v_{j}$ if $\mu\left(p_{i}\right)=q_{j}$, and $u_{i}=0$ if $\mu\left(p_{i}\right)=q_{0}$.

Among the set of stable outcomes, two particularly interesting outcomes can be highlighted.

Definition 8- The payoff $(\underline{u}, \bar{v})$ is called the seller-optimal stable payoff if $\bar{v} \geq v$ and $\underline{u}$ $\leq u$ for all stable payoffs $(u, v)$.

If $\mu$ is compatible with $(\underline{u}, \bar{v})$ we say that the outcome $(\underline{u}, \bar{v} ; \mu)$ is the seller-optimal stable outcome with the matching $\mu$. Similarly we can define the buyer-optimal stable payoff.

The following well-known results from Shapley and Shubik (1972) will be stated here without proof. ${ }^{5}$

Proposition 1- Every buyer-seller market $M$ has at least one stable outcome.

Consequently, the core and the set of competitive equilibria are non-empty sets.

Proposition 2- If $\mu$ is an optimal matching, then it is compatible with any stable payoff.

Thus, if $\mu$ is an optimal matching, then it is competitive for any competitive equilibrium.

Proposition 3- If $(u, v ; \mu)$ is a stable outcome, then $\mu$ is an optimal matching.

Consequently if $(v, \mu)$ is a competitive equilibrium then $\mu$ is an optimal matching. Moreover, according to Propositions 2 and 3, the set of stable outcomes is the Cartesian product of the set of stable payoffs and the set of optimal matchings.

Proposition 4- Every buyer-seller market $M$ has a seller-optimal stable payoff and a buyer-optimal stable payoff. 
The existence of a seller-optimal stable payoff is equivalent to the statement that there is a unique vector of equilibrium prices, $\bar{v}$, that is optimal for the sellers, in the sense that $\bar{v}_{j} \geq v_{j}$ for all $q_{j}$ in $Q$ and for all equilibrium price vector $v$. Similar statement applies to the buyer-optimal stable payoff. The equilibrium price vector $\bar{v}$ is called the maximum equilibrium price vector and a competitive equilibrium $(\bar{v} ; \mu)$ is called a maximum competitive equilibrium.

\section{3- THE BUYER-SELLER SEQUENTIAL MECHANISM}

Our main purpose in this paper is to analyze the outcome of a simple selling and buying procedure that will be denoted by $H^{\sigma}$. We attempt to propose a mechanism as simple as possible, at the same time have it produce directly both a matching between sellers and buyers and prices for the objects sold. It is a two-phase mechanism. In the first phase, each seller proposes a tentative price for his object. Proposals are made simultaneously. In the second phase buyers, sequentially, decide which object to buy, if any. The tentative prices of sold objects become actual prices paid by buyers to sellers, while sellers of unsold objects keep their objects and receive nothing.

Formally, let $\sigma$ be a permutation of the set of buyers (i.e., $\sigma$ represents a line). The mechanism $H^{\sigma}$ works as follows:

(i) First, sellers play simultaneously. A strategy for seller $q_{j}$ consists of choosing a price $v_{j} \in R_{+}$for his object. We consider that the null object $q_{0}$ is always available at the price $v_{0}=0$.

(ii) Second, buyers play sequentially, following the order given by $\sigma$. To describe the buyers' strategies, denote by $Q_{i}$ the set of all objects which are still available for $p_{i}$ when she is called to play under the ordering $\sigma$ (notice that $q_{0} \in Q_{i}$ ). A strategy for buyer $p_{i}$ is a function that selects an element of $Q_{i}$, for each vector of offers $v$.

5 See also Roth and Sotomayor (1990) for an exposition of these results. 
Following the mechanisms, it is not possible for two different buyers to select the same object, unless the object is $q_{0}$. Then, the joint strategies for the buyers, one for each of them, produces a feasible matching $\mu$, where $\mu\left(p_{i}\right)$ is the object of $Q_{i}$ chosen by $p_{i}$. Any non-selected object will be unmatched under $\mu$. The mechanism allocates the objects according to the matching $\mu$. Also, every buyer $p_{i}$ pays $v_{j}$ to seller $q_{j}$ if $\mu\left(p_{i}\right)=q_{j}$; hence seller $q_{j}$ receives $v_{j}$ if $q_{j}$ is sold and receives nothing if $q_{j}$ is unsold. That is, $H^{\sigma}(v$, $\mu)=\left(v^{*}, \mu\right)$, where $v^{*}$ is the vector of prices actually paid in the market, which constitutes the payoff vector of the sellers: $v_{j}^{*}=v_{j}$ if $\mu\left(q_{j}\right) \in P$ and $v^{*}{ }_{j}=0$ if $q_{j}$ is unsold. Sometimes we will use the notation $H_{j}^{\sigma}(v, \mu)$ for seller $q_{j}$ 's payoff, $v^{*}$. Moreover, the payoff of buyer $p_{i}$ is then $u_{i}=\alpha_{i j}-v^{*}{ }_{j}=\alpha_{i j}-v_{j}$ when $\mu\left(p_{i}\right)=q_{j}$. Clearly, $u_{i}=0$ if $\mu\left(p_{i}\right)=$ $q_{0}$.

Given some permutation $\sigma$, we are going to consider the subgame perfect equilibria in pure strategies $(\sigma-\mathrm{SPE})$ of the game induced by the mechanism $H^{\sigma}$. The set of best responses for the buyers to the sellers' joint strategies, say $v$, is the set of SPE of the game that starts once $v$ has been decided. (Notice that the elements of this set are matchings). For these equilibria, it is always the case that each buyer chooses, once the prices $v$ have been selected, one among the best objects available for her (this includes the possibility of her selecting the null object). In other words, each buyer $p_{i}$ chooses an object in $D_{i}\left(v \mid Q_{i}\right)$, which is the set of objects in $Q_{i}$ that maximize $p_{i}^{\prime}$ s utility payoff. That is,

$$
D_{i}\left(v \mid Q_{i}\right) \equiv\left\{q_{j} \in Q_{i} ; \alpha_{i j}-v_{j} \geq \alpha_{i h}-v_{h}, \text { for all } q_{h} \text { in } Q_{i}\right\}
$$

A matching $\mu$ obtained in this way is called $\sigma$-competitive for the prices $v$. Formally:

Definition 9 - Given the permutation $\sigma$ and the feasible price vector $v$, the matching $\mu$ is $\sigma$-competitive for $v$ if every buyer $p_{i}$ chooses $\mu\left(p_{i}\right) \in D_{i}\left(v \mid Q_{i}\right)$.

Therefore, $\mu$ is $\sigma$-competitive for $v$ if each buyer $p_{i}$ chooses one among her best responses to $v$ and to the actions of the previous buyers. For some price vectors, there are several $\sigma$-competitive matchings. This happens when a buyer must select one out of 
a set of several objects among which she is completely indifferent. Even if her action does not change her final utility, it strongly influences the utilities of the sellers coming after her. To illustrate this situation, consider the following example:

Example 1- Consider a set of objects $Q=\left\{q_{0}, q_{1}, q_{2}, q_{3}\right\}$ and a set of buyers $P=\left\{p_{1}\right.$, $\left.p_{2}, p_{3}\right\}$. Let the matrix $\alpha$ be such that $\alpha_{11}=\alpha_{12}=\alpha_{21}=\alpha_{23}=\alpha_{32}=\alpha_{33}=2$ and the other entries are zero. Let $\sigma=\left(p_{1}, p_{2}, p_{3}\right)$. Suppose that the sellers choose the vector of prices $v=(0,1,1,1)$ and that the buyers choose $\mu\left(p_{1}\right)=q_{2}, \mu\left(p_{2}\right)=q_{3}$ and $\mu\left(p_{3}\right)=q_{0}$. Each buyer is selecting a best response given the prices of the objects and the actions of the previous buyers. However, notice that if either $p_{1}$ or $p_{2}$ chooses $q_{1}$ then buyer $p_{3}$ can select either $q_{2}$ or $q_{3}$, respectively. If this happens, buyers $p_{1}$ and $p_{2}$ have the same utility payoff as before but $p_{3}$ is strictly better off.

Sometimes, it is reasonable to assume that if a buyer is completely indifferent among several actions, but one of them leads to a more efficient outcome for the buyers coming after her, then she will choose this action. When we restrict attention to such strategies we say that buyers are selecting $\sigma$-maximal matchings. Formally we have:

Definition 10- Given the permutation $\sigma$ and the price vector $v \in R^{n}$, we say that $\mu$ is $\sigma$ maximal for $v$ if $\mu$ is $\sigma$-competitive for $v$ and it is Pareto-efficient for the buyers in the set of $\sigma$-competitive matchings for $v$. That is, let $u$ be the payoff vector of the buyers associated to $(v, \mu)$. Then $\mu$ is $\sigma$-maximal for $v$ if and only if (a) $\mu$ is $\sigma$-competitive for $v$, and (b) for any matching $\mu^{\prime}$ which is $\sigma$-competitive for $v$, if $u_{i}<u_{i}^{\prime}$ for some $p_{i}$ in $P$, then $u_{k}>u^{\prime}{ }_{k}$ for some $p_{k}$ in $P$, where $u$ is the payoff vector of the buyers associated to $\left(v, \mu^{\prime}\right)$.

We must point out that given $\sigma$, if $\nu$ is an equilibrium price and $\mu$ is an optimal matching then $\mu$ is $\sigma$-maximal for $v$. However, the converse is not true. In fact, consider, for example, a market with only one object $q_{1}$ and only one buyer $p_{1}$. The buyer is indifferent between buying the object at the price $v=\alpha_{11}$ or not buying it. Any of her options is a $\sigma$-maximal matching for $v$, but only the first one is an optimal matching. 
Let us now look at the possible strategic actions by the sellers. To analyze whether a vector of prices is part of an equilibrium, we must check that no seller is interested in deviating from his proposed price. Sometimes, a deviation by a seller is profitable or not depending on the expected reaction by the buyers. The following example illustrates this:

Example 2- Consider a set of objects $Q=\left\{q_{0}, q_{1}, q_{2}, q_{3}\right\}$ and a set of buyers $P=\left\{p_{1}\right.$, $\left.p_{2}, p_{3}\right\}$. Let the matrix $\alpha$ be such that $\alpha_{11}=1, \alpha_{12}=\alpha_{22}=\alpha_{23}=2, \alpha_{33}=1$ and the other entries are zero. Let $\sigma=\left(p_{2}, p_{1}, p_{3}\right)$. Suppose that the sellers choose the vector of prices $v=(0,0,0,0)$. If $q_{1}$ increases his price from $v_{1}=0$ to $v_{1}^{\prime}=0.5$, this deviation can be profitable for $q_{1}$ if $p_{2}$ buys $q_{2}$, for then $p_{1}$ will buy $q_{1}$. However, the deviation is not profitable if $p_{2}$ buys $q_{3}$. In this case the best response for $p_{1}$ is to buy $q_{2}$ and $p_{3}$ will be unmatched. Observe that the set of best responses for the buyers has only these two matchings and both of them are $\sigma$-maximal (under the first matching the payoff vector of the buyers is $(0.5,2,1)$ and under the second one is $(2,2,0))$.

What behavior can be predicted for $q_{1}$ in this game? We will assume that a seller who analyses the possibility of deviating takes an optimistic view. That is, a seller changes his strategy whenever he has a chance to be better off. Therefore we are looking for seller strategies under which no seller has a chance to be better off. This means that we are interested in equilibria in the strong version. (A similar concept has been defined by Demange and Gale (1985).) Also, to avoid inconsistencies, we continue to require that the buyers use $\sigma$-maximal strategies. The formal definition of a $\sigma$-SPE in the strong sense is then the following:

Definition 11- Let $\sigma$ be some permutation, $v \in R^{n}{ }_{+}$a price vector and $\mu$ some $\sigma$-maximal matching for $v$. We say that $(v, \mu)$ is a $\sigma-S P E$ in the strong sense if for no $q_{j}$ there is a $v^{\prime}$, with $v_{h}^{\prime}=v_{h}$ for $q_{h} \neq q_{j}$, and a $\sigma$-maximal matching $\mu^{\prime}$ for $v^{\prime}$, such that $H_{j}^{\sigma}\left(v^{\prime}, \mu^{\prime}\right)>$ $H_{j}^{\sigma}(v, \mu)$.

Notice that by considering only the equilibria in the strong sense we restrict the set of SPE. 


\section{4- THE SET OF EQUILIBRIA}

In this section we show that, if the buyers always choose $\sigma$-maximal matchings, the set of outcomes of the equilibria in the strong sense coincides with the set of maximum competitive equilibria for any $\sigma$. That is, first, the final prices paid by the buyers at equilibrium correspond to the maximum equilibrium price vector for the assigned objects. Second, the assignment of objects to buyers corresponds to an optimal matching (and every optimal matching is part of some $\sigma$-SPE). The previous result implies, in particular, that the vectors of sellers' payoff and buyers' utility are the same under any $\sigma$-SPE in the strong sense. Moreover, in equilibrium, the ordering determined by $\sigma$ is irrelevant.

In this section, we will denote the $\sigma$-SPE in the strong sense as $\sigma$-SPE, for short. When $(v, \mu)$ is a $\sigma$-SPE we will say that $v$ is part of a $\sigma$-SPE and that $H^{\sigma}(v, \mu)$ is the $\sigma$ SPE outcome.

To characterize the set of $\sigma$-SPE outcomes of $H^{\sigma}$, we will use Proposition 5 below, which is an immediate consequence of Hall's theorem (see Gale (1960)). To state it, we need the following definition:

Definition 12- Let $v \in R^{n}{ }_{+}$, and $P^{\prime} \subseteq P$ be such that $q_{0} \notin D_{i}(v)$ for all $p_{i} \in P^{\prime}$. We say that $D$ $\equiv \cup_{p_{i} \in P} D_{i}(v)$ is an overdemanded set under $v$ if $|D|<\left|P^{\prime}\right|^{6}$

That is, a set $D$ is overdemanded if the number of buyers demanding only objects in $D$ is greater than the number of objects in $D$.

Proposition 5- (Corollary of Hall's Theorem)- Let $v \in R^{n}{ }_{+}$. A competitive matching for $v$ exists if and only if there is no overdemanded set under $v$.

6 Given a set $A$, we denote its cardinality by $|A|$. 
We start our analysis of the $\sigma$-SPE of the mechanism $H^{\sigma}$ by stating a result that will allow us to use Hall's theorem. Proposition 6 shows that, at a $\sigma$-SPE, there exists no overdemanded set.

Proposition 6- Let $v$ be the vector of strategies of the sellers in a $\sigma$-SPE. Then, there is no overdemanded set under $v$.

In the proof of Proposition 6, we will use Lemma 1, whose proof is relegated to the Appendix. It establishes the following property. Consider a price vector which is part of a $\sigma$-SPE, and a group of buyers that obtain a strictly positive payoff and that buy objects in their demand sets. Then, there is some object which is not bought by any of the buyers in this group but which belongs to some of their demand sets.

Lemma 1- Let $v$ be the vector of strategies of the sellers in a $\sigma$-SPE. Let $\mu$ be some $\sigma$ maximal matching for $v$ such that $\mu\left(p_{i}\right) \in D_{i}(v)$ for all $p_{i} \in P^{\prime} \subseteq P$ and denote by $u$ the payoff vector of the buyers associated with $(v, \mu)$. Suppose that $u_{i}>0$ for all $p_{i} \in P^{\prime} \subseteq P$. Let $Q^{\prime} \equiv \mu\left(P^{\prime}\right)$. Then, there exists some $p_{i} \in P^{\prime}$ and $q_{j} \notin Q^{\prime}$ such that $q_{j} \in D_{i}(v)$.

Proof of Proposition 6- Let $\sigma=\left(p_{1}, p_{2}, \ldots, p_{m}\right)$ and denote $P^{r} \equiv\left\{p_{1}, \ldots, p_{r}\right\}$. For the proof of the proposition, it is sufficient to prove that for all $1 \leq r \leq m$, and all $P^{\prime} \subseteq P^{r}$ with $q_{0} \notin D_{i}(v)$ for every $p_{i} \in P^{\prime}$, we have that $\left|\cup_{p_{i} \in P^{P}} D_{i}(v)\right| \geq\left|P^{\prime}\right|$. We will prove it by induction on $r$. If $r=1$ it is obvious.

Suppose that for all $P^{\prime} \subseteq P^{r-1}$, with $q_{0} \notin D_{i}(v)$ for every $p_{i} \in P^{\prime}$, we have that $\left|P^{\prime}\right| \leq\left|\cup_{p_{i} \in P} D_{i}(v)\right|$. Before proving that the property also holds for $r$, we show first that there exists a $\sigma$-maximal matching $\mu^{\prime}$ for $v$ such that $\mu^{\prime}\left(p_{i}\right) \in D_{i}(v)$ for all $i=1$, $2, \ldots, r-1$. That is, we want to show that there is a $\sigma$-maximal matching $\mu^{\prime}$ for $M=(P$, $Q, \alpha)$, whose restriction to $M^{\prime}=\left(P^{r-1}, Q, \alpha^{\prime}\right)$, is a competitive matching for $v$, where $\alpha^{\prime}$ is the restriction of $\alpha$ to $P^{r-1} \times Q$. Then, let $S=\left\{\mu ; \mu\right.$ is $\sigma$-competitive for $v$ in $M$ and $\mu\left(p_{i}\right) \in D_{i}(v)$ for all $\left.i=1,2, \ldots, r-1\right\}$. 
That is, $S$ is the set of all $\sigma$-competitive matchings for $v$ whose restriction to $M^{\prime}$ is competitive. By the induction hypothesis there is no overdemanded set of objects in $M^{\prime}$ under $v$. Proposition 5 implies that there exists some competitive matching for $v$ in $M^{\prime}$. Therefore the set $S$ is non empty, since the matching given by Proposition 5 can be easily extended to a $\sigma$-competitive matching for $v$ (we only need to take a best response for the buyers from $p_{r}$ on, which always exists). Since $S$ is non-empty and finite, there is at least a matching $\mu^{\prime} \in S$ which is Pareto-efficient for the buyers among all matchings in $S$. We claim that $\mu^{\prime}$ is a $\sigma$-maximal matching for $v$. That is, $\mu^{\prime}$ is Pareto-efficient for the buyers not only among all matchings in $S$, but also among all $\sigma$-competitive matchings. In fact, if $\mu^{\prime}$ is not $\sigma$-maximal then there is a $\sigma$-competitive matching $\mu$ such that $u_{i} \geq u_{i}^{\prime}$ for all $p_{i} \in P$, with strict inequality holding for at least one buyer, where $u^{\prime}$ and $u$ are the payoff vectors of the buyers associated with $\left(v, \mu^{\prime}\right)$ and $(v, \mu)$, respectively. However, it is necessarily the case that $u_{i}=u_{i}^{\prime}$ for all $p_{i} \in P^{r-1}$, since all these players are maximizing their utility payoff under $\mu^{\prime}$. Hence $\mu \in S$, which contradicts the assumption that $\mu^{\prime}$ is Pareto-efficient for the buyers.

We now prove that the property holds for $r$ by contradiction. Suppose that there is some $P^{\prime} \subseteq P^{r}$ with $q_{0} \notin D_{i}(v)$ for every $p_{i} \in P^{\prime}$, and such that $\left|P^{\prime}\right|>\left|\cup_{p_{i} \in P} D_{i}(v)\right|$. Let $D \equiv$ $\cup_{p_{i} \in P} D_{i}(v)$. It follows by the induction hypothesis that $P^{\prime} \not \subset P^{r-1}$, so $p_{r} \in P^{\prime}$. Also, applying the induction hypothesis to $P^{\prime}-\left\{p_{r}\right\} \subseteq P^{r-1}$, it is the case that $\left|P^{\prime}\right|-1=\left|P^{\prime}-\left\{p_{r}\right\}\right| \leq|D|$ $<\left|P^{\prime}\right|-1$. Denoting $P^{*} \equiv P^{\prime}-\left\{p_{r}\right\}$, the previous inequalities imply that $|D|=\left|P^{*}\right|$ and $D=\cup_{p_{i} \in P^{*}} D_{i}(v)$. Moreover, since the matching $\mu^{\prime} \in \mathrm{S}$ (that we found previously) is competitive for $v$ in $M^{\prime}=\left(P^{r-1}, Q, \alpha^{\prime}\right)$ and $P^{*} \subseteq P^{r-1}$, then $\mu^{\prime}\left(p_{i}\right) \in D_{i}(v)$ for all $p_{i} \in P^{*}$. Thus, it is necessarily the case that $D=\mu^{\prime}\left(P^{*}\right)$.

Now use Lemma 1 to obtain that there exists some $p_{\mathrm{i}} \in P^{*}$ and $q_{\mathrm{j}} \notin D$ such that $q_{\mathrm{j}} \in D_{\mathrm{i}}(v)$, which is a contradiction. Q.E.D.

Our first theorem asserts that the $\sigma$-SPE outcomes are always competitive equilibria. The insight obtained from Proposition 6 is very useful for both the understanding and the proof of Theorem 1 . 
Theorem 1- Let $(v, \mu)$ be a $\sigma$-SPE. Then, $H^{\sigma}(v, \mu)$ is a competitive equilibrium.

Proof- By Proposition 6, there is no overdemanded set of objects at the prices $v$. Therefore, Proposition 5 guarantees that there is a matching $\mu^{\prime}$ which is a competitive matching for $v$. Now observe that the buyers maximize their utility payoffs under $\mu^{\prime}$ and $\mu$ is $\sigma$-maximal. Then $v$ is a competitive price with matching $\mu$.

To prove that $H^{\sigma}(v, \mu)=\left(v^{*}, \mu\right)$ is a competitive equilibrium, we have to show that $v^{*}$ is a competitive price vector with matching $\mu$, where $v^{*}{ }_{j}=v_{j}$ if $q_{j}$ is sold and $v^{*}{ }_{j}=0$ otherwise. Denote by $u$ the payoff vector of the buyers associated to $(v, \mu)$. Since there are no $\mu$-expensive objects under $v^{*}$, proving the theorem only requires us to show that if $q_{j}$ is unsold and $v_{j}>v_{j}^{*}=0$, then $u_{i} \geq \alpha_{i j}-v^{*}{ }_{j}$ for all $p_{i} \in P$. Suppose, by way of contradiction, that there exists some unsold object, say $q_{j}$, such that $v_{j}>v^{*}{ }_{j}=0$ and $u_{i}<$ $\alpha_{i j}-v_{j}^{*}$ for some $p_{i} \in P$. There are some $\lambda>0$ and $\gamma>0$ such that $u_{i}+\lambda=\alpha_{i j}-\gamma$. Let $v^{\prime}$ be such that $v_{j}^{\prime}=\gamma$, and $v_{h}^{\prime}=v_{h}$ if $q_{h} \neq q_{j}$. Since $v$ is part of a $\sigma$-SPE, it follows that $q_{j}$ is unsold at any $\sigma$-maximal matching for $v^{\prime}$. However, $\max _{h \neq j} \alpha_{i h}-v_{h}=u_{i}<u_{i}+\lambda=\alpha_{i j}-v_{j}^{\prime}$, hence buyer $p_{i}$ is not playing her best response at any $\sigma$-maximal matching for $v^{\prime}$, which is a contradiction. Therefore, $H^{\sigma}(v, \mu)$ is a competitive equilibrium. Q.E.D.

Theorem 1 ensures that only competitive equilibria are candidates for a $\sigma$-SPE of the mechanism. Theorem 2 goes a step further: Aside from the cases in which a seller has an object unsold but sets an expensive price on his object, only the maximum equilibrium prices can be part of a $\sigma$-SPE of the mechanism $H^{\sigma}$.

Theorem 2- Let $(v, \mu)$ be a $\sigma$-SPE. Then, $H^{\sigma}(v, \mu)=(\bar{v}, \mu)$, where $\bar{v}$ is the maximum equilibrium price vector.

To prove the theorem, we will use the following lemma, whose proof is developed in the Appendix. 
Lemma 2- Let $\left(v^{1}, \mu^{1}\right)$ be a $\sigma$-SPE and set $H^{\sigma}\left(v^{1}, \mu^{1}\right) \equiv\left(v^{*}, \mu^{1}\right)$. Let $\left(u^{2}, v^{2}, \mu^{2}\right)$ be some feasible outcome and $Q^{+}=\left\{q_{j} \in Q ; v_{j}{ }_{j}>v^{*}{ }_{j}\right\}$. If $Q^{+} \neq \varnothing$, then there exists some pair $\left(p_{i}, q_{h}\right) \in P \times Q$ such that $u_{i}^{2}+v^{2}{ }_{h}<\alpha_{i h}$.

Proof of Theorem 2- Let $H^{\sigma}(v, \mu)=\left(v^{*}, \mu\right)$. We have to show that $v^{*} \geq v^{\prime}$ for all equilibrium prices $v^{\prime}$. Let $v^{\prime}$ be some equilibrium price vector, $\mu^{\prime}$ a competitive matching for $v^{\prime}$, and $u^{\prime}$ the payoff vector of the buyers associated with $\left(v^{\prime}, \mu^{\prime}\right)$. Denote

$Q^{+}=\left\{q_{j} \in Q ; \mu^{\prime}\left(q_{j}\right) \in P\right.$ and $\left.v_{j}^{\prime}>v_{j}^{*}\right\}$.

If $Q^{+} \neq \varnothing$, then Lemma 2 asserts that there is some pair $\left(p_{i}, q_{h}\right) \in P \times Q$ such that $u_{i}^{\prime}+v_{h}^{\prime}<$ $\alpha_{i h}$, which is impossible since the price vector $v^{\prime}$ is competitive. Therefore $Q^{+}=\varnothing$. Since there are no expensive objects at any equilibrium prices it follows that $v^{*} \geq v^{\prime}$. That is, $v^{*}=\bar{v} . \quad$ Q.E.D.

It easily follows from Theorem 2 that if $(v, \mu)$ is a $\sigma$-SPE and $v_{j}=0$ for all unmatched seller $q_{j}$, then $v$ is the maximum equilibrium price vector. Of course, any prices $v^{\prime}$, with $v_{j}^{\prime}=v_{j}$ if $q_{j}$ is matched under $\mu$, and $v_{j}^{\prime} \geq v_{j}$ if $q_{j}$ is unmatched under $\mu$, is also part of a $\sigma$ SPE leading to the maximum competitive equilibrium.

From Theorem 2 we know that $H^{\sigma}$ must necessarily lead to a maximum competitive equilibrium. We now show that it is indeed the case that every maximum competitive equilibrium is a $\sigma$-SPE.

Theorem 3- Let $(\underline{u}, \bar{v} ; \mu)$ be a seller-optimal stable outcome. Then, $(\bar{v}, \mu)$ is a $\sigma$-SPE for any $\sigma$.

This theorem is proved with the help of Lemma 3. We include the proof of the lemma in the Appendix.

Lemma 3- Let $(\underline{u}, \bar{v})$ be the seller-optimal stable payoff. Let $\mu$ be an optimal matching. Construct a graph whose vertices are $P \cup Q$ and with two types of arcs. If $\mu\left(p_{i}\right)=q_{j}$ there is an arc from $q_{j}$ to $p_{i}$; if $q_{j} \in D_{i}(\bar{v})$ and $q_{j} \neq \mu\left(p_{i}\right)$ there is an arc from $p_{i}$ to $q_{j}$. Let 
$p_{k} \in P$ with $\underline{u}_{k}>0$. Then, there exists an oriented path starting at $p_{k}$ and ending at an unsold object or at a buyer with a zero payoff.

Proof of Theorem 3- Consider any permutation $\sigma$. The outcome of the strategies $(\bar{v}$, $\mu)$ is $H^{\sigma}(\bar{v}, \mu)=(\bar{v} ; \mu)$. Since $\mu$ is competitive for $\bar{v}$, it is the case that $\mu$ is $\sigma$-maximal for $\bar{v}$. Hence, to prove the theorem, it is sufficient to show that $\bar{v}$ is indeed an equilibrium for the sellers. Let $q_{j} \in Q-\left\{q_{0}\right\}$. We are going to show that $q_{j}$ will be unsold at any $\sigma$-maximal matching for $v^{\prime}$, where $v_{j}^{\prime}>\bar{v}_{j}$ and $v_{h}^{\prime}=\bar{v}_{h}$ if $q_{h} \neq q_{j}$. However, before we proceed to prove this property, we show that there is some competitive matching for $\bar{v}$, say $\mu^{\prime}$, which leaves $q_{j}$ unsold. The cases to be considered are the following:

Case 1. $q_{j}$ is unmatched at $\mu$. Then, take $\mu^{\prime}=\mu$.

Case 2. $\mu\left(q_{j}\right)=p_{i}$ and $\underline{u}_{i}=0$. In this case, take $\mu^{\prime}$ so that $\mu^{\prime}$ agrees with $\mu$ on the choices of the buyers other than $p_{i}$ and associate $p_{i}$ to the null object.

Case 3. $\mu\left(q_{j}\right)=p_{i}$ and $\underline{u}_{i}>0$. By Lemma 3, there exists an oriented path $c$ starting at $p_{i}$ and ending at an unsold object $q_{s}$ or at a buyer $p_{s}$ with payoff zero. Since $c$ does not cycle then $q_{j}$ is not in $c$. Set $c \equiv\left(p=p_{1}, q_{1}, p_{2}, q_{2}, \ldots, p_{\mathrm{s}}, q_{\mathrm{s}}\right)$ or $c \equiv\left(p=p_{1}, q_{1}, p_{2}, q_{2}, \ldots\right.$, $\left.p_{s}, q_{s}, p_{s+1}\right)$. Now consider the matching $\mu^{\prime}$ that matches $p_{t}$ to $q_{t}$, for all $t=1,2, \ldots, s$, that leaves $p_{s+1}$ unmatched if $p_{s+1}$ is on the path, that otherwise agrees with $\mu$ with regard to every object in $Q-\left\{q_{j}\right\}$ and every buyer in $P$ that are not on the path, and that sets $\mu^{\prime}\left(q_{j}\right)$ $=q_{j}$. Every buyer obtains the same utility under $\mu^{\prime}$ as under $\mu$, since $\mu^{\prime}\left(p_{t}\right)=q_{t} \in D_{t}(v)$, for all $t=1,2, \ldots, s$, and $\mu^{\prime}$ agrees with $\mu$ for the other buyers. Therefore, $\mu^{\prime}$ is a competitive matching for $\bar{v}$.

In all of the three cases, we have found a matching $\mu^{\prime}$ for $\bar{v}$ under which $q_{j}$ is not sold and such that every buyer maximizes her utility payoff under $\bar{v}$. Therefore, under $\mu^{\prime}$ every buyer $p_{k}$ will be maximizing her utility payoff also for the price vector $v^{\prime}$, and she obtains a utility of $\underline{u}_{k}$. Then, we claim that $q_{j}$ will be unsold at any $\sigma$-maximal matching for $v^{\prime}$ : if $q_{j}$ was sold at the price $v_{j}^{\prime}$ to some $p_{k}$, we would have that $\alpha_{k j}-v_{j}^{\prime}<\alpha_{k j}-\bar{v}_{j} \leq \underline{u}_{k}$, 
while the utility of the other buyers can not be higher than $\underline{u}$, so the matching could not be $\sigma$-maximal. Q.E.D.

Theorem 2 states that the only outcomes that can be reached through the mechanism $H^{\sigma}$ are maximum competitive equilibria if we use equilibria in the strong sense. Theorem 3 asserts that any maximum equilibrium price vector is part of a $\sigma$-SPE. As a consequence, the mechanism $H^{\sigma}$ implements in $\sigma$-SPE the set of maximum competitive equilibria.

Corollary 1- Let $\sigma$ be a permutation of the buyers. Then, $H^{\sigma}$ implements in $\sigma$-SPE the set of maximum competitive equilibria.

Proof- Immediate from theorems 2 and 3.

Since any $\sigma$-SPE in the strong sense is a $\sigma$-SPE in the weak sense we have:

Corollary 2- Let $(\underline{u}, \bar{v} ; \mu)$ be a seller-optimal stable outcome. Then, $(\bar{v}, \mu)$ is a $\sigma$-SPE in the weak sense for any $\sigma$.

\section{5- DISCUSSION OF THE $\sigma$-MAXIMAL STRATEGIES}

In our analysis of the proposed selling procedure, we have assumed that buyers use $\sigma$-maximal strategies. An indifferent buyer picks an object that leads to an efficient outcome for the buyers coming after her. This requires each buyer to know the preferences of all the buyers that follow her in the line. There are some environments in which this may be reasonable. However, there are also other situations in which this hypothesis is demanding and, in those environments, one may argue that the mechanism is not very practical.

How can buyers chose a $\sigma$-maximal strategy in those cases where the information about the others' preferences is not complete? Are there reasonable ways through which the buyers can actually follow $\sigma$-maximal strategies if, a priori, they do not know each other preferences? We claim that such reasonable ways exist. 
It is often the case that when buyers are in a queue, they chat about their respective choices. The buyers that are not well placed in the line may try to make their preferences clear so that the buyers ahead in the queue solve indifferences in their favor. This can be seen as an informal way of transmitting information. If the number of buyers is large and there is no possibility for mutual knowledge or communication among buyers, the informal channel may be difficult to implement. In this case, we would need an external referee that would ask for the buyers' preferences and, once the buyers reveal them, chooses a $\sigma$-maximal matching taking into account the reports by the buyers and the predetermined order $\sigma$.

The two previous mechanisms, the informal and the formal one, are quite similar. For simplicity, let us consider the formal mechanism in which a referee is called in to arbitrate. The mechanism is a useful way to transmit information only if buyers do have incentives to declare their preferences truthfully. A simple and easy test about the strategic incentives to lie is to check whether truth-telling is a max-min strategy. Following a max-min strategy, a buyer maximizes the minimum level of utility she can guarantee for herself independently of the others' preferences. Indeed, in the previous mechanism, truth-telling is a max-min strategy. The reason is that, by telling the truth, the $i$-th buyer obtains, at least, her $i$-th most preferred object, and this is the most she can guarantee for herself. ${ }^{7}$ If a buyer does not have any information about the others' preferences, following a max-min strategy is "safe". Therefore, truth-telling is a reasonable strategy.

There is also a less demanding approach to the problem of the transmission of information among buyers that allows obtaining our implementation result. We are going to develop this approach in some detail. We propose a mechanism in which a buyer is not asked to reveal her preferences, as in the previous proposal, but she only has to reveal her indifferences to the following buyer in the line. Informally, once the

\footnotetext{
7 Truth telling is not the only max-min strategy. Every strategy in which the $i$-th buyer keeps the same set of the $i$ most preferred objects but changes the order of these objects is also a max-min strategy.
} 
first buyer realizes that she is indifferent among a certain set of objects, she says so to the second buyer. The second buyer can also tell the third buyer about the different paths (that is, the different matchings for buyers 1 and 2) among which she is indifferent, respecting the will of the first buyer, and so on. We will refer to this mechanism as mechanism $R$. In the rest of the section, we will first formally define the mechanism $R$ and will show that buyers do not have any incentives to lie when they play it. Then, we will characterize the set of matchings that can be expected to be formed following the mechanism $R$. Finally, we will extend the implementation result to the framework in which buyers form such matchings.

We describe the mechanism $R$. It is played once the sellers have posted the vector of prices $v$. For notational simplicity, we consider $\sigma=\left(p_{1}, \ldots, p_{m}\right)$.

(1) At $t=1$, buyer $p_{1}$ chooses a non-empty subset $S_{1}$ of the set of feasible matchings for $M_{1} \equiv\left(\left\{p_{1}\right\}, Q, \alpha\right)$. For $p_{1}$, choosing a set $S_{1}$ is equivalent to choosing a subset of $Q$.

(2) At $t=2$, buyer $p_{2}$ chooses a non-empty subset $S_{2}$ of the set of feasible matchings for $M_{2} \equiv\left(\left\{p_{1}, p_{2}\right\}, Q, \alpha\right)$ whose restriction to $M_{1}$ is an element of $S_{1}$. For $p_{2}$, choosing a set $S_{2}$ amounts to telling to $p_{1}$ the object(s) $p_{2}$ wants $p_{1}$ to pick up among the "objects" in $S_{1}$ and then, for each of those objects, choosing one or more objects for herself.

( $t$ ) At $t$, for $2<t \leq m$, buyer $p_{t}$ chooses a non-empty subset $S_{t}$ of the set of feasible matchings for $M_{t} \equiv\left(\left\{p_{1}, \ldots, p_{t}\right\}, Q, \alpha\right)$ whose restriction to $M_{t-1}$ is an element of $S_{t-1}$. That is, at $t$, the elements of $S_{t}$ are matchings for buyer $p_{t}$ and for the buyers previous to her. Buyer $p_{t}$ has, however, to respect the will of the previous buyers, expressed in $S_{t-1}$.

After all buyers have played, we have a final set $S_{m}$. The matching that will actually take place will be chosen at random among the matchings in $S_{m}$.

Proposition 7- In the mechanism $R$, the following is a SPE for a vector of prices $v$ : given a set $S_{t-1}$, buyer $p_{t}$ reports a set $S_{t}$ that consists in her most preferred matchings (given the price vector $v$ ) among the matchings whose restriction to $M_{t-1}$ is in $S_{t-1}$. 
Proof- When $p_{t}$ chooses $S_{t}$, she knows that she will be buying according to one of the paths in $S_{t}$, independently on the choices of the buyers coming after her. A buyer does not have any incentives to lie at all and truth-telling is a best response to every possible set $S_{t-1}$. Hence, truth-telling is a SPE. Q.E.D.

The important fact about the proposed strategy (truth-telling) is that a buyer does not need to know the other buyers' preferences. The set of possible outcomes of a buyer does depend on the previous buyers' preferences, but her strategy is independent on them. On the other hand, the preferences of the buyers coming after her do not have any influence on the choice of a buyer. This is the case because, following the strategy, buyer $p_{t}$ is indifferent between the objects that the matchings in $S_{t}$ assign to her.

We now show that, when the buyers declare their true indifferences, that is, when they follow the proposed SPE, then they end up forming a $\sigma$-maximal matching. In fact, they always form a particular type of matching; we will call them top matchings. A top matching is a $\sigma$-maximal matching that is one of the most preferred by the buyers when they can choose according to $\sigma$. That is, a top matching is one of the most preferred $\sigma$ maximal matchings for the second buyer; it is one of the most preferred for the third buyer among the $\sigma$-maximal matchings most preferred by the second buyer; and so on. More formally:

Definition 13- A feasible matching $\mu$ for $M$ is a top matching if it is $\sigma$-maximal and, for any other $\sigma$-maximal matching $\mu^{\prime}$ such that $u_{i}^{\prime}>u_{i}$ for some buyer $p_{i}$, there exists a buyer $p_{k}$, with $k<i$, such that $u_{k}>u_{k}^{\prime}$, where $u$ and $u^{\prime}$ are the payoff vectors of the buyers associated to $(v, \mu)$ and $\left(v, \mu^{\prime}\right)$ respectively.

Proposition 8- If buyers declare truthfully their indifferences in the mechanism $R$ (that is, they use the SPE strategies proposed in Proposition 7), then $S_{m}$ is the set of top matchings.

Proof- Take $\mu \in S_{m}$. Given that buyers choose their best matchings, $\mu$ is $\sigma$-competitive for $v$. We prove that $\mu$ is $\sigma$-maximal and that it is a top matching if we show that, for 
any other $\sigma$-competitive matching $\mu^{\prime}$ for $v$ such that $u_{i}^{\prime}>u_{i}$ for some buyer $p_{i}$, there exists a buyer $p_{k}$, with $k<i$, such that $u_{k}>u_{k}^{\prime}$, where $u$ and $u^{\prime}$ are the payoff vectors of the buyers associated to $(\nu, \mu)$ and $\left(v, \mu^{\prime}\right)$ respectively. We make the proof by contradiction. Suppose that there is a $\sigma$-competitive matching $\mu^{\prime}$ for $v$ such that $u_{i}^{\prime}>u_{i}$ for some buyer $p_{i}$ and $u_{k}=u_{k}^{\prime}$ for every $k<i$. (Note that $u_{1}=u_{1}^{\prime}$, since $\mu$ and $\mu^{\prime}$ are both $\sigma$-competitive matchings.) Since $\mu$ and $\mu^{\prime}$ provide the same utility to buyers $p_{1}, \ldots, p_{i-1}$, it is easy to see that the restriction of $\mu$ to $M_{k}$ is in $S_{k}$ if and only if the restriction of $\mu^{\prime}$ to $M_{k}$ is in $S_{k}$, for every $k<i$. Since $\mu \in S_{m}$, it is the case that the restriction of $\mu$ to $M_{i-1}$ is in $S_{i-1}$. Therefore, the restriction of $\mu^{\prime}$ to $M_{i-1}$ is also in $S_{i-1}$. But then, buyer $p_{i}$ is not maximizing her utility by choosing a set $S_{i}$ that includes the restriction of $\mu$ to $M_{i}$ : she would obtain a higher utility by taking $S_{i}^{\prime}=$ \{restriction of $\mu^{\prime}$ to $M_{i}$ \}. Hence $S_{m}$ is contained in the set of top matchings.

It remains to show that every top matching is in $S_{m}$. Note first that $S_{m}$ is non-empty by construction. Take $\mu$ in $S_{m}$ and let $\mu$ ' be any other top matching. Then both matching are also $\sigma$-maximal. Let $u$ and $u$ ' be the payoff vectors of the buyers associated to $(v, \mu)$ and $\left(v, \mu^{\prime}\right)$, respectively. By Definition 13 we must have that $u_{1}=u^{\prime}{ }_{1}$. Now apply the definition of a top matching to both matchings to get that $u_{i}=u^{\prime}{ }_{i}$ for all $i=1, \ldots, m$. Finally, given that every player is indifferent between $\mu$ and $\mu$, then the restriction of $\mu$ to $M_{k}$ is in $S_{k}$ if and only if the restriction of $\mu^{\prime}$ to $M_{k}$ is in $S_{k}$. Therefore, $\mu^{\prime} \in S_{m}$. Q.E.D.

Proposition 8 tells us what matchings can be expected if each buyer follows the equilibrium strategy of declaring truthfully her indifferences to the next buyer in the line. On the one hand, only $\sigma$-maximal matchings are expected. On the other hand, only a subset of the set of $\sigma$-maximal matchings (the top matchings) will be formed. This can be problematic because, to prove the implementation result in theorems 2 and 3, we have assumed that buyers can use any $\sigma$-maximal strategy. What happens if we assume that buyers choose top strategies (strategies leading to top matchings) with certainty? (For example, because they use the proposed communication channel to break 
indifferences in favor of the buyers after them in the line.) Next theorem shows that, in this framework, the implementation result still holds.

Theorem 4- Let $\sigma$ be a permutation of the buyers. Then, the set of SPE in the strong sense of the mechanism $H^{\sigma}$ coincides with the set of maximum competitive equilibria when the buyers use top strategies.

The main message of Theorem 4 (whose proof is relegated to the Appendix) is that it is possible to relax the hypothesis of perfect information among buyers and still obtain the implementation result. If buyers do not know each other preferences, they can set up a simple channel to transmit the relevant information (their indifferences). Theorem 4 shows that when the buyers use such a channel, the final prices are still the maximum competitive prices and the final matching is optimal.

\section{6- SOME ADDITIONAL EXAMPLES}

In order to obtain our results, we have restricted the analysis to what we have called maximal strategies by the buyers (or top strategies, in Section 5), and to equilibria in the strong sense by the sellers. We show here, through examples, that without such restrictions the implementation result (Corollary 1) no longer holds. In this section, the notation $\sigma$-SPE* stands for subgame perfect equilibria in pure strategies in general, without restricting attention to equilibria in the strong sense and/or allowing any strategy by the buyers.

The first question is what happens if we still restrict attention to $\sigma$-SPE* in the strong sense, while allowing the buyers to use any $\sigma$-competitive strategies. Note that this change diminishes the set of equilibria since the optimistic seller looking for a deviation considers as possible a larger set of buyers' strategies. Example 3 shows that the set of equilibria may be empty for all $\sigma$. In the example, we will use the following result, whose proof is relegated to an Appendix.

Proposition 9- If $(v, \mu)$ is a $\sigma-\mathrm{SPE}^{*}$ in the strong sense then $H^{\sigma}(v, \mu)$ is a competitive equilibrium. Consequently $\mu$ is a $\sigma$-maximal matching for $v$. 
Example 3- (The set of $\sigma$-SPNE* may be empty for all $\sigma$ ) Consider a set of objects $Q=\left\{q_{0}, q_{1}, q_{2}, q_{3}\right\}$ and a set of buyers $P=\left\{p_{1}, p_{2}, p_{3}\right\}$. Let $\alpha$ be such that $\alpha_{11}=\alpha_{33}=5$, $\alpha_{12}=\alpha_{32}=1, \alpha_{13}=\alpha_{21}=\alpha_{23}=\alpha_{31}=4$ and $\alpha_{22}=0$. There is only one stable payoff in this market: $u=(1,0,1)$ and $v=(0,4,0,4)$. There are four optimal matchings, denoted $\mu_{k}, k=1,2,3,4: \mu_{1}\left(p_{1}\right)=q_{1}, \mu_{1}\left(p_{2}\right)=q_{2}, \mu_{1}\left(p_{3}\right)=q_{3} ; \mu_{2}\left(p_{1}\right)=q_{1}, \mu_{2}\left(p_{2}\right)=q_{3}, \mu_{2}\left(p_{3}\right)=$ $q_{2} ; \mu_{3}\left(p_{1}\right)=q_{2}, \mu_{3}\left(p_{2}\right)=q_{1}, \mu_{3}\left(p_{3}\right)=q_{3} ;$ and $\mu_{4}\left(p_{1}\right)=q_{1}, \mu_{4}\left(p_{2}\right)=q_{0}, \mu_{4}\left(p_{3}\right)=q_{3}$. Then, by Proposition $7,\left(v, \mu_{k}\right), k=1,2,3,4$ are the only candidates for a $\sigma$-SPE* in the strong sense, for any $\sigma$. However, we show now that none of them are $\sigma$-SPE*s in the strong sense for any $\sigma$.

Consider first $\sigma=\left(p_{1}, p_{2}, p_{3}\right)$ or $\sigma=\left(p_{2}, p_{3}, p_{1}\right)$. The strategies $\left(v, \mu_{i}\right)$ do not constitute a $\sigma$-SPE* in the strong sense because the deviation $v_{2}^{\prime}=0.5$ followed by the $\sigma$-competitive matching $\mu_{2}$ for the first ordering and by $\mu_{3}$ for the second one is profitable to seller $q_{2}$. Second, if $\sigma=\left(p_{3}, p_{1}, p_{2}\right)$ or $\sigma=\left(p_{3}, p_{2}, p_{1}\right)$, then if seller $q_{1}$ deviates with $v_{1}^{\prime}=4.5$, the $\mu_{2}$ is also a best response for the buyers. Thus the deviation $v_{1}^{\prime}$ is profitable to $q_{1}$. Finally, if $\sigma=\left(p_{2}, p_{1}, p_{3}\right)$ or $\sigma=\left(p_{1}, p_{3}, p_{2}\right)$, then the deviation $v_{3}^{\prime}$ $=4.5$ is profitable to $q_{3}$ if the buyers use the $\sigma$-competitive strategy $\mu_{3}$. Also in these cases $\left(v, \mu_{i}\right)$ is not a $\sigma$-SPE* in the strong sense. Note that $\mu_{2}$ or $\mu_{3}$ are not $\sigma$-maximal matchings for $v^{\prime}$ in none of the cases (this is only a confirmation of Theorem 3 )

The second question is whether restricting attention to equilibria in the strong sense is actually a restriction (note that the set of equilibria is a superset of the set of equilibria in the strong sense). When buyers only use maximal strategies, Example 4 shows that it is indeed the case that there exist equilibria different from the maximum competitive equilibria.

Example 4- Consider a set of objects $Q=\left\{q_{0}, q_{1}, q_{2}, q_{3}, q_{4}\right\}$, a set of buyers $P=\left\{p_{1}\right.$, $\left.p_{2}, p_{3}, p_{4}\right\}$, and $\sigma=\left(p_{1}, p_{2}, p_{3}, p_{4}\right)$. Let $\alpha_{14}=\alpha_{23}=\alpha_{31}=\alpha_{34}=\alpha_{42}=\alpha_{43}=0$ and let the other entries be equal to 2 . The maximum price vector is $\bar{v}=(0,2,2,2,2)$ and an optimal matching is $\mu: \mu\left(p_{1}\right)=q_{1}, \mu\left(p_{2}\right)=q_{2}, \mu\left(p_{3}\right)=q_{3}, \mu\left(p_{4}\right)=q_{4}$. However, we claim that $v=(0,1,1,1,1)$ followed by the $\sigma$-maximal matching $\mu$ is part of a $\sigma$-SPE* (in the 
weak sense) in which the out-of-equilibrium $\sigma$-maximal strategies for the buyers are the following:

(a) If $v_{1}^{\prime}>v_{1}$ and $v_{i}^{\prime}=v_{i}$, for $i=2,3,4$, then $\mu_{1}\left(p_{1}\right)=q_{3}, \mu_{1}\left(p_{2}\right)=q_{2}, \mu_{1}\left(p_{3}\right)=q_{0}, \mu_{1}\left(p_{4}\right)=$ $q_{4}$.

(b) If $v_{2}^{\prime}>v_{2}$ and $v_{i}^{\prime}=v_{i}$, for $i=1,3,4$, then $\mu_{2}\left(p_{1}\right)=q_{1}, \mu_{2}\left(p_{2}\right)=q_{4}, \mu_{2}\left(p_{3}\right)=q_{3}, \mu_{2}\left(p_{4}\right)=$ $q_{0}$.

(c) If $v_{3}^{\prime}>v_{3}$ and $v_{i}^{\prime}=v_{i}$, for $i=1,2,4$, then $\mu_{3}\left(p_{1}\right)=q_{1}, \mu_{3}\left(p_{2}\right)=q_{4}, \mu_{3}\left(p_{3}\right)=q_{2}, \mu_{3}\left(p_{4}\right)=$ $q_{0}$.

(d) If $v_{4}^{\prime}>v_{4}$ and $v_{i}^{\prime}=v_{i}$, for $i=1,2,3$, then $\mu_{4}\left(p_{1}\right)=q_{3}, \mu_{4}\left(p_{2}\right)=q_{2}, \mu_{4}\left(p_{3}\right)=q_{0}, \mu_{4}\left(p_{4}\right)=$ $q_{1}$.

To check that the strategies are $\sigma$-maximal, take for example case (a). If $\mu_{1}$ is not $\sigma$ maximal, then it is necessarily the case that $\mu^{\prime}\left(p_{3}\right) \in\left\{q_{1}, q_{2}, q_{3}\right\}$, for any $\mu^{\prime}$ Paretosuperior for the buyers to $\mu_{1}$. But in this case either $p_{1}$, or $p_{2}$, or $p_{4}$ are strictly worse-off with $\mu^{\prime}$ than with $\mu_{1}$, so $\mu^{\prime}$ is not Pareto-superior to $\mu_{1}$.

We may also find competitive equilibria that are not a $\sigma$-SPE*.

Example 5- Consider $Q=\left\{q_{1}\right\}, P=\left\{p_{1}, p_{2}\right\}$ and $\sigma=\left(p_{1}, p_{2}\right)$. Let $\alpha$ be such that $\alpha_{11}$ $=8$ and $\alpha_{21}=7$. The competitive equilibrium $(v=7, \mu)$, where $\mu\left(p_{1}\right)=q_{1}$, is not a $\sigma$ SPE* for any $\sigma$. In fact, the deviation $v_{1}^{\prime}=7.5$ is surely profitable to $q_{1}$.

Our final question is what happens if we look for $\sigma$-SPE* without restricting attention either to maximal strategies or to equilibria in the strong sense. The following example shows that the $\sigma$-SPE* are not necessarily competitive equilibria.

Example 6- Consider $Q=\left\{q_{0}, q_{1}, q_{2}, q_{3}, q_{4}\right\}, P=\left\{p_{1}, p_{2}, p_{3}\right\}$ and $\sigma=\left(p_{1}, p_{2}, p_{3}\right)$. Let $\alpha$ be such that $\alpha_{11}=\alpha_{22}=5, \alpha_{13}=\alpha_{24}=3, \alpha_{31}=\alpha_{32}=7$, and the other entries are equal to zero. The price vector $v=(0,2,2,0,0)$ with the matching $\mu\left(p_{1}\right)=q_{1}, \mu\left(p_{2}\right)=$ 
$q_{2}, \mu\left(p_{3}\right)=q_{0}$, is not a competitive equilibrium. However, we claim that they constitute a $\sigma$-SPE* with the following out-of-equilibrium continuation:

(a) If $v_{1}^{\prime}>v_{1}$ and $v_{i}^{\prime}=v_{i}$, for $i=2,3,4$, then $\mu_{1}\left(p_{1}\right)=q_{3}, \mu_{1}\left(p_{2}\right)=q_{4}, \mu_{1}\left(p_{3}\right)=q_{2}$.

(b) If $v_{2}^{\prime}>v_{2}$ and $v_{i}^{\prime}=v_{i}$, for $i=1,3,4$, then $\mu_{2}\left(p_{1}\right)=q_{3}, \mu_{2}\left(p_{2}\right)=q_{4}, \mu_{2}\left(p_{3}\right)=q_{1}$.

(e) If $v^{\prime}$ different from the previous (a)-(b), then take any $\sigma$-competitive strategy.

\section{7- CONCLUSION}

In our opinion, the analysis developed in this paper shows that a mechanism in which both buyers and sellers use very simple strategies can work well. The mechanism is meant to reflect the working of some markets. Each seller posts the price for his object, price that will become final if the object is sold. Each buyer chooses an object (if any) to buy, the choices by the buyers directly producing the final matching in the market. We have shown that, when buyers use maximal strategies, the strategies played by buyers and sellers at any subgame perfect equilibria in the strong sense lead to the maximum equilibrium price vector and to an optimal matching. Therefore, the partnerships formed between buyers and sellers are efficient and the actual prices constitute a competitive equilibrium in this market (the competitive equilibrium most preferred by the sellers). Moreover, in equilibrium, the order in which the buyers are in the line is not relevant: the set of equilibria is independent of the line (the permutation $\sigma$ ) and the utility that each buyer and seller obtain at any equilibrium is the same.

In the mechanism that we propose, the sellers post prices first and the actual matching is then chosen by the buyers sequentially. This timing seems the most natural one. On the one hand, if we interchange the roles so that buyers propose prices and sellers choose the matching, the buyers' strategies are more complex: since objects are different from the buyers' perspective, each buyer needs to set a vector of prices, instead of just one price. This makes the mechanism less attractive and the analysis more difficult. Note, however, that the result seems very sensitive to the side of the market that moves first. The ability of fixing the prices causes that the equilibrium prices are the 
highest among the possible competitive prices. On the other hand, one can also think of a mechanism in which the buyers choose simultaneously their preferred objects once the sellers have simultaneously proposed prices. This is in the spirit of the mechanisms analyzed in Alcalde, Pérez-Castrillo, and Romero-Medina (1998) and Alcalde and Romero-Medina (1999). However, in contrast with the previous papers, the implementation of the mechanism in the assignment games brings about the problem of deciding what happens if two different buyers choose the same object. The sequentiality of our mechanism allows us to avoid the use of tie-breaking rules, as well as the strong implicit coordination needed for the equilibria of simultaneous mechanisms. 


\section{REFERENCES}

Alcalde, J., 1996, "Implementation of Stable Solutions to Marriage Problems," Journal of Economic Theory 69, 240-254.

Alcalde, J., D. Pérez-Castrillo and A. Romero-Medina, 1998, "Hiring Procedures to Implement Stable Allocations," Journal of Economic Theory 82, 469-480.

Alcalde, J. and A. Romero-Medina, 1999, "Simple Mechanisms to Implement the Core of College Admissions Problems," forthcoming in Games and Economic Behavior.

Crawford, V.P. and E.M. Knoer, 1981, "Job Matching with Heterogeneous Firms and Workers," Econometrica 49, 437-450.

Demange., G. and D. Gale, 1985, "The Strategy of Two-sided Matching Markets," Econometrica 53, 873-988.

Demange., G., D. Gale and M. Sotomayor, 1986, "Multi-Item Auctions," Journal of Political Economy 94, 863-872.

Dubins, L.E. and D.A. Freedman, 1981, "Machiavelli and the Gale-Shapley Algorithm," American Mathematical Monthly 88, 485-494.

Gale, D., 1960, The Theory of Linear Economic Models, McGraw Hill, New York.

Gale, D. and L1.S. Shapley, 1962, "College Admissions and the Stability of Marriage," American Mathematical Monthly 69, 9-15.

Gale, D. and M. Sotomayor, 1985-a, "Some Remarks on the Stable Matching Problem," Discrete Applied Mathematics, 11, 223-232.

Gale, D. and M. Sotomayor, 1985-b, "Ms. Machiavelli and the Stable Matching Problem," American Mathematical Monthly, 92, 261-268. 
Kamecke, U., 1989, "Non-Cooperative Matching Games," International Journal of Game Theory, 18, 423-431.

Kelso, A.S. and V.P. Crawford, 1982, "Job Matching, Coalition Formation, and Gross Substitutes," Econometrica 50, 1483-1504.

Pérez-Castrillo, D., 1994, "Cooperative Outcomes through Non-Cooperative Games," Games and Economic Behavior 7, 428-440.

Perry, M., and P. Reny, 1994, "A Non-Cooperative View of Coalition Formation and the Core," Econometrica 62, 795-817.

Roth, A.E., 1982, "The Economics of Matching: Stability and Incentives," Mathematics of Operations Research 7, 617-628.

Roth, A.E., 1984, "Misrepresentation and Stability in the Marriage Problem," Journal of Economic Theory 34, 383-387.

Roth, A.E. and M. Sotomayor, 1990, Two-Sided Matching: A Study in Game Theoretic Modeling and Analysis, Econometric Society Monograph Series, Cambridge University Press, New York.

Serrano, R., 1995, "A Market to Implement the Core," Journal of Economic Theory 67, 285-294.

Shapley, Ll.S. and M. Shubik, 1972, "The Assignment Game I: The Core," International Journal of Game Theory 1, 111-130. 


\section{APPENDIX}

Proof of Lemma 1- Suppose, by way of contradiction, that for all $p_{i} \in P^{\prime}$ and all $q_{j} \notin Q^{\prime}$ we have that $q_{j} \notin D_{i}(v)$. That is, suppose that $Q^{\prime} \equiv \mu\left(P^{\prime}\right)=\cup_{p_{i} \in P^{\prime}} D_{i}(v)$. Since $u_{i}>0$ for all $p_{i} \in P^{\prime}$, it implies that $q_{0} \notin Q^{\prime}$ and so

$$
\left|P^{\prime}\right|=\left|Q^{\prime}\right|
$$

Let $p_{k}$ be the last buyer in $P^{\prime}$ under $\sigma$ and $q_{h}=\mu\left(p_{k}\right)$ be the object that $p_{k}$ buys. From $D_{k}(v) \subseteq Q^{\prime}$ it follows that $u_{k}>\alpha_{k j}-v_{j}$ for all $q_{j} \notin Q^{\prime}$. Now use that $u_{k}>0$ to get that there exists some $\lambda>0$ such that

$$
\begin{gathered}
u_{k}-\lambda>\alpha_{k j}-v_{j} \text { for all } q_{j} \notin Q^{\prime}, \text { and } \\
u_{k}-\lambda>0 .
\end{gathered}
$$

Let $v^{\prime}$ be such that $v^{\prime}{ }_{h}=v_{h}+\lambda, v_{j}^{\prime}=v_{j}$ for all $q_{j} \neq q_{h}$. Since $p_{k}$ is the last buyer in $P$ ' under $\sigma$, it follows that $p_{k}$ will buy $q_{h}$ at the price $v_{h}$ if the previous buyers keep buying according to $\mu$. This is immediate from (1) and (2). Indeed, equation (1) implies that $q_{h}$ is the unique available object belonging to $Q^{\prime}$ when $p_{k}$ is called to play; equation (2) implies that $\left\{q_{h}\right\}=D_{k}\left(v^{\prime} \mid Q_{k}\right)$. Hence $\mu$ is $\sigma$-competitive for $v^{\prime}$.

We will show that $\mu$ is $\sigma$-maximal for $v$, which will contradict the initial hypothesis that $v$ is part of a $\sigma$-SPE. Again, we do the proof by contradiction. Suppose that $\mu$ is not $\sigma$-maximal for $v^{\prime}$. Then, there is some matching $\mu$ ' which is $\sigma$-competitive for $v$ ' and such that:

$$
u_{i}^{\prime} \geq u_{i} \text { for all } p_{i} \in P-\left\{p_{k}\right\} \text { and } u_{k}^{\prime} \geq u_{k}-\lambda \text {, }
$$

with at least one strict inequality, where $u$ ' is the payoff vector for the buyers associated to $\left(v^{\prime}, \mu^{\prime}\right)$. From (4) and (2) it follows that

$$
u_{k}^{\prime}>\alpha_{k j}-v_{j} \text { for all } q_{j} \notin Q^{\prime} \text {. }
$$


However, using the hypothesis that $\mu\left(p_{i}\right) \in D_{i}(v)$ for all $p_{i} \in P^{\prime}$ and (4), it follows that for all $p_{i} \in P^{\prime}-\left\{p_{k}\right\}, u_{i}^{\prime}=u_{i}\left(u_{i}^{\prime}>u_{i}\right.$ is not possible because $p_{i}$ is maximizing her payoff under $\mu)$. Consequently $\mu^{\prime}\left(p_{i}\right) \in D_{i}(v)$ for all $p_{i} \in P^{\prime}-\left\{p_{k}\right\}$, and so $\mu^{\prime}\left(p_{i}\right) \in Q^{\prime}$ for all $p_{i} \in P^{\prime}-\left\{p_{k}\right\}$. Also if $p_{i} \neq p_{k}$ then $\mu^{\prime}\left(p_{i}\right) \neq q_{h}$, for if not $\alpha_{i h}-v_{h}>\alpha_{i h}-v_{h}-\lambda=u_{i}^{\prime}=u_{i} \geq$ $\alpha_{i h}-v_{h}$, which is a contradiction. Therefore

$$
\mu^{\prime}\left(p_{i}\right) \in Q^{\prime}-\left\{q_{h}\right\} \text { for all } p_{i} \in P^{\prime}-\left\{p_{k}\right\} \text {. }
$$

By (3) and (4), we obtain that $u^{\prime}{ }_{k}>0$, so $p_{k}$ is matched under $\mu^{\prime}$. That $\mu^{\prime}\left(p_{k}\right) \in Q^{\prime}$ follows from (5). Now use (1) and (6) to get that $\mu^{\prime}\left(p_{k}\right)=q_{h}$.

Since $\lambda$ can be taken arbitrarily small, it is then easily seen that if $\mu$ ' is $\sigma$-competitive for $v^{\prime}$ then $\mu^{\prime}$ is also $\sigma$-competitive for $v$. Moreover, $u_{i}^{\prime} \geq u_{i}$ for all $p_{i} \in P$ and $u_{i}>u_{i}$ for some $p_{i} \in P\left(p_{i} \in P-P^{\prime}\right)$, which contradicts the $\sigma$-maximality of $\mu$ for $v$. Hence, $\mu$ is $\sigma$ maximal for $v^{\prime}$. Therefore, there is a profitable deviation from $v$, which is a contradiction. Then, there exists some $p_{i} \in P^{\prime}$ and $q_{j} \notin Q^{\prime}$ such that $q_{j} \in D_{i}(v)$. Q.E.D.

Proof of Lemma 2- Case 1. $\mu^{1}\left(Q^{+}\right) \neq \mu^{2}\left(Q^{+}\right)$. Since every seller in $Q^{+}$is matched by $\mu^{2}$, choose $p_{i} \in \mu^{2}\left(Q^{+}\right)-\mu^{1}\left(Q^{+}\right)$, say $p_{i}=\mu^{2}\left(q_{j}\right)$. Denote by $u^{1}$ the payoff vector of the buyers associated with $\left(v^{1}, \mu^{1}\right)$. By Theorem $1,\left(u^{1}, v^{*} ; \mu^{1}\right)$ is stable. It then follows that $0 \leq u^{2}{ }_{i}$ $<u_{i}^{1}$, since otherwise $\alpha_{i j}=u_{i}^{2}+v_{j}^{2}>u_{i}^{1}+v^{*}{ }_{j}$, which would contradict the stability of $\left(u^{1}, v^{*}, \mu^{1}\right)$. This implies that $p_{i}$ is necessarily matched under $\mu^{1}$, say $p_{i}=\mu^{1}\left(q_{h}\right)$, where $q_{h} \notin Q^{+}$(i.e., $v^{*}{ }_{h} \geq v^{2}{ }_{h}$ ). Then, $\alpha_{i h}=u_{i}^{1}+v^{*}{ }_{h}>u_{i}^{2}{ }+v_{h}^{2}$, which proves the lemma.

Case 2. $\mu^{2}\left(Q^{+}\right)=\mu^{1}\left(Q^{+}\right)$. Consider any $p_{i} \in \mu^{2}\left(Q^{+}\right)=\mu^{1}\left(Q^{+}\right)$and denote $q_{j}=\mu^{1}\left(p_{i}\right) \in Q^{+}$ and $q_{k}=\mu^{2}\left(p_{i}\right) \in Q^{+}$. First, $u_{i}^{1}=\alpha_{i j}-v^{*}{ }_{j} \geq \alpha_{i k}-v^{*}{ }_{k}$ since $\left(u^{1}, v^{*} ; \mu^{1}\right)$ is stable by Theorem 1. Second, $v^{*}{ }_{k}<v^{2}{ }_{k}$ because $q_{k} \in Q^{+}$. Then $u_{i}^{1}>\alpha_{i k}-v^{2}{ }_{k}=u^{2}{ }_{i} \geq 0$. Therefore $u_{i}{ }_{i}>u_{i}^{2} \geq 0$ for all $p_{i} \in \mu^{2}\left(Q^{+}\right)$. Moreover, stability implies that $\mu^{1}\left(p_{i}\right) \in D_{i}\left(v^{1}\right)$ for all $p_{i} \in P$. By Lemma 1, making $Q^{\prime} \equiv Q^{+}$and $P^{\prime} \equiv \mu^{1}\left(Q^{+}\right)$, there exists some $p_{i} \in \mu^{1}\left(Q^{+}\right)$and $q_{h} \notin Q^{+}$such that $q_{h} \in D_{i}\left(v^{1}\right)$. Hence $\alpha_{i h}=u_{i}^{1}+v^{*}{ }_{h}>u_{i}^{2}+v^{*}{ }_{h} \geq u_{i}^{2}+v^{2}{ }_{h}$, where the last inequality comes from $q_{h} \notin Q^{+}$, and the result follows. Q.E.D. 
Proof of Lemma 3- Suppose that there is no such a path and denote by $S$ and $T$ the sets of objects and buyers, respectively, that can be reached from $p_{k}$. Then $\underline{u}_{i}>0$ for all $p_{i} \in T$ and each object in $S$ is sold to some buyer in $T$. Furthermore, if $q \notin S$, then there is no buyer in $T$ who demands $q_{j}$ at prices $\bar{v}$. In this case, it is possible to decrease $\underline{u}_{i}$ for all $p_{i}$ in $T$ by some $\lambda>0$ and increase $\bar{v}_{j}$ for all $q_{j} \in S \cup\left\{\mu\left(p_{k}\right)\right\}$ by the same $\lambda>0$ and still have a stable outcome, which contradicts the maximality of $\bar{v}$. Q.E.D.

Proof of Theorem 4- Lemma 1 still holds if we replace the following sentence in its statement: "Let $\mu$ be some $\sigma$-maximal matching for $v . . . "$ with: "Let $\mu$ be some top matching for $v$..." For the proof, see that $\mu$ is also a top matching for $v$, since every buyer $p_{t}$ is matched under $\mu$ to some object in $D_{i}(v)$. For the proof of Proposition 6, apply the mechanism $R$ and obtain any top matching (instead of simply a $\sigma$-maximal matching) whose restriction to $M^{\prime}=\left(P^{r-1}, Q, \alpha\right)$ is a competitive matching for $v$ in $M^{\prime}$. This is possible because Proposition 5 implies that there exists some competitive matching for $v$ in $M^{\prime}$, so every buyer in $\left\{p_{1}, \ldots, p_{r-1}\right\}$ is maximizing her payoff under this matching. Now, apply Lemma 1 and get the desired result.

Since Proposition 6 holds, Theorem 1 also holds in this framework. Lemma 1 and Theorem 1 are used in the proof of Lemma 2, which is needed in the proof of Theorem 2. Lemma 3 does not change. For the proof of Theorem 3 observe that $\mu$ is a top matching for $\bar{v}$ and keep the rest of the proof unchanged. Therefore, Theorem 3 and Corollary 1 still hold in this new set-up. Q.E.D.

Proof of Proposition 9- Remember that $(v, \mu)$ is a $\sigma-S P E^{*}$ in the strong sense if for no $q_{j}$ there is a $v^{\prime}$, with $v_{h}^{\prime}=v_{h}$ for $q_{h} \neq q_{j}$, and a $\sigma$-competitive matching $\mu^{\prime}$ for $v^{\prime}$, such that $H_{j}^{\sigma}\left(v^{\prime}, \mu^{\prime}\right)>H_{j}^{\sigma}(v, \mu)$. Let $H^{\sigma}(v, \mu)=\left(v^{*}, \mu\right)$. Suppose by way of contradiction that $\left(v^{*}\right.$, $\mu$ ) is not a competitive equilibrium. Let $u$ be the payoff vector of the buyers associated to $\left(v^{*}, \mu\right)$. Then there is a pair $\left(p_{i}, q_{j}\right)$ such that $u_{i}+v^{*}{ }_{j}<\alpha_{i j}$. Either (a) $q_{j}$ is a $\mu$ expensive object for $v\left(q_{j}\right.$ is unsold and $\left.v_{j}>v^{*}{ }_{j}=0\right)$, or (b) $q_{j}$ was sold at price $v^{*}{ }_{j}=v_{j}$ with $\sigma\left(\mu\left(q_{j}\right)\right)<\sigma\left(p_{i}\right)$. In this case, choose $q_{j}$ so that

$$
\text { if } u_{i}+v_{h}^{\prime}<\alpha_{i h} \text { then } \sigma\left(\mu\left(q_{h}\right)\right)<\sigma\left(\mu\left(q_{j}\right)\right) \text {. }
$$


Let $\lambda>0$ and $\gamma>0$ be such that $\left(u_{i}+\lambda\right)+\left(v_{j}^{*}+\gamma\right)=\alpha_{i j}$ and let $v^{\prime}$ be such that $v_{j}^{\prime}=v_{j}^{*}+\gamma$ and $v_{h}^{\prime}=v_{h}$ for all $q_{h} \neq q_{j}$. Then $\alpha_{i j}-v_{j}^{\prime}=u_{i}+\lambda>u_{i} \geq \alpha_{i h}-v^{*}{ }_{h}$, so

$$
\alpha_{i j}-v_{j}^{\prime}>\alpha_{i h}-v^{*}{ }_{h} \text { for all } q_{h} \in Q_{i}
$$

where $Q_{i}$ is the set of available objects for $p_{i}$ under $\mu$. In case (a) let every buyer $p_{k}$, with $\sigma\left(p_{k}\right)<\sigma\left(p_{i}\right)$, choose $\mu\left(p_{k}\right)$, which is still a best response for $p_{k}$ to $v^{\prime}$. Thus the set of available objects for $p_{i}$ under $v^{\prime}$ is still $Q_{i}$. Hence, by (8), $q_{j}$ will be the only object in the demand set of $p_{i}$ at prices $v^{\prime}$. Therefore $q_{j}$ will be sold to $p_{i}$. In this case seller $q_{j}$ wins by deviating, which contradicts the fact that $(v, \mu)$ is a $\sigma$-SPE*.

Consider now case (b). Let every buyer $p_{k}$ with $\sigma\left(p_{k}\right)<\sigma\left(\mu\left(q_{j}\right)\right)$ play $\mu\left(p_{k}\right)$, which is still a best response for $p_{k}$ to $v^{\prime}$. Then at the time $p_{i}$ is called to play, if $q_{j}$ is still available, no matter which were the choice of $\mu\left(q_{j}\right)$ and the choices of the buyers who came after $\mu\left(q_{j}\right), q_{j}$ will be the $p_{i}$ 's most preferred object in her set of available objects. In fact, if $\alpha_{i h}-v_{h}^{\prime}>\alpha_{i j}-v_{j}^{\prime}=u_{i}+\lambda>u_{i}$ then $u_{i}+v_{h_{h}}<\alpha_{i h}$, so $\sigma\left(\mu\left(q_{h}\right)\right)<\sigma\left(\mu\left(q_{j}\right)\right)$ by (7). By hypothesis this implies that $\mu\left(q_{h}\right)$ buys $q_{h}$ at $v^{\prime}$, so $q_{h}$ is not available to $p_{i}$ when she comes to play. Now use (2) to get that $p_{i}$ will buy $q_{j}$ at $v_{j}^{\prime}$. Therefore, in any case, $v_{j}^{\prime}$ is a profitable deviation. Hence $(v, \mu)$ is a competitive equilibrium. Moreover, as a consequence of this result, $\mu$ is an optimal matching so it is a $\sigma$-maximal matching for $v$ and the proof is complete. Q.E.D. 